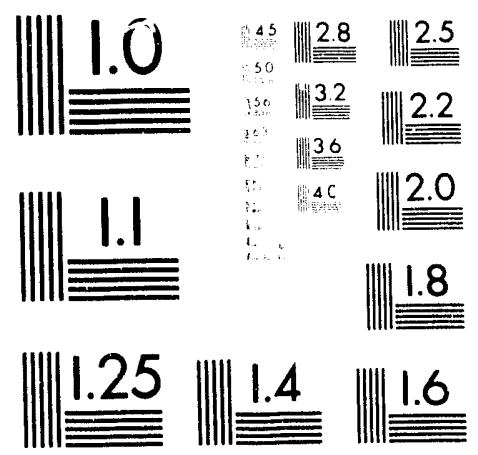



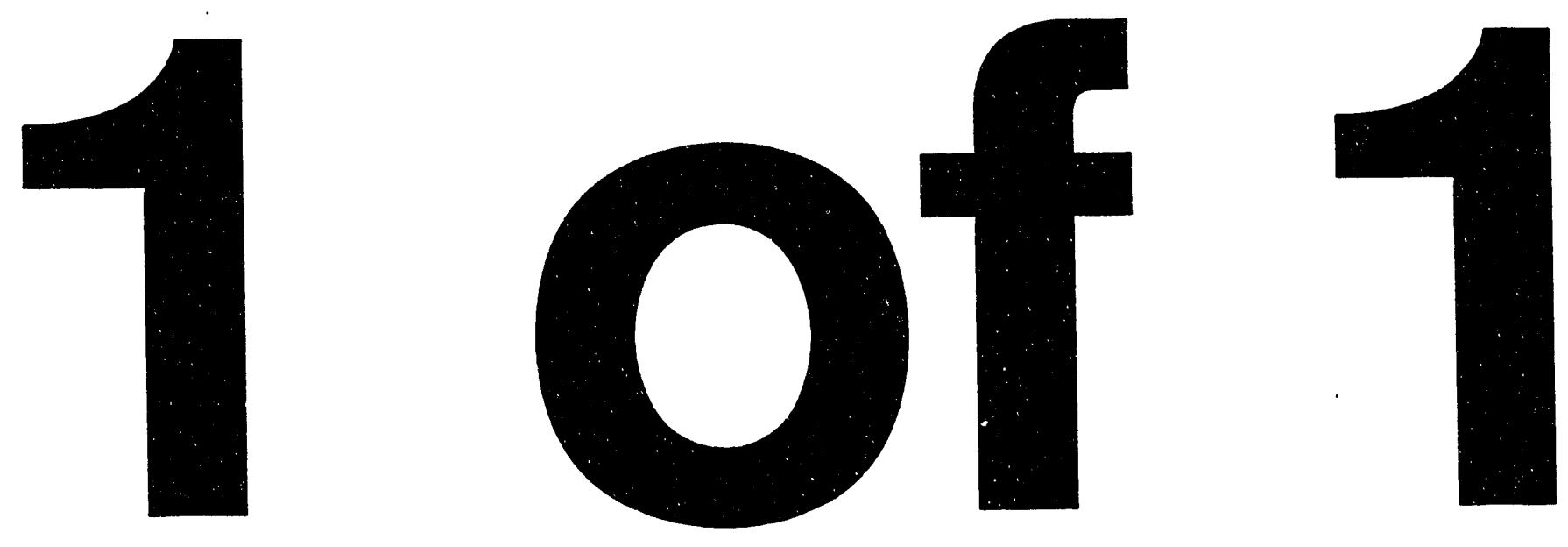


\section{INSTITUTE FOR \\ FUSION STUDIES}

DOE/ET-53088-617

IFSR \#617

Shear Flow Generation by Reynolds Stress and $\triangleleft 27$

Suppression of Resistive $g$-Modes

H. Sugama

National Institute for Fusion Science

Nagoya, 464-01, Japan

and

W. HORTON

Institute for Fusion Studies

The University of Texas at Austin

Austin, Texas 78712

August 1993

THEUNIVERSITY OF TEXAS 


\title{
Shear Flow Generation by Reynolds Stress and Suppression of Resistive $g$-Modes
}

\author{
H. Sugama \\ National Institute for Fusion Science \\ Nagoya 464-01, Japan \\ and \\ W. Horton \\ Institute for Fusion Studies \\ The University of Texas at Austin \\ Austin, Texas 78712
}

\begin{abstract}
Suppression of resistive $g$-mode turbulence by background shear flow generated from a small external flow source and amplified by the fluctuation-induced Reynolds stress is demonstrated and analyzed. The model leads to a paradigm for the low-to-high ( L-H) confinement mode transition. To demonstrate the L-H transition model, singlehelicity nonlinear fluid simulations using the vorticity equation for the electrostatic potential, the pressure fluctuation equation and the background poloidal flow equation are used in the sheared slab configuration. The relative efficiency of the external flow and the Reynolds stress for producing shear flow depends on the poloidal flow damping parameter $\nu$ which is given by neoclassical theory. For large $\nu$, the external flow is a dominant contribution to the total background poloidal shear flow and its strength predicted by the neoclassical theory is not enough to suppress the turbulence significantly. In contrast, for small $\nu$, we show that the fluctuations drive a Reynolds stress that becomes large and suddenly, at some critical point in time, shear flow
\end{abstract}


much larger than the external flow is generated and leads to an abrupt, order unity reduction of the turbulent transport just like that of the L-H transition in tokamak experiments. It is also found that, even in the case of no external flow, the shear flow generation due to the Reynolds stress occurs through the nonlinear interaction of the resistive $g$-modes and reduces the transport. To supplement the numerical solutions we derive the Landau equation for the mode amplituda of the resistive $g$-mode taking into account the fluctuation-induced shear flow and analyze the opposite action of the Reynolds stress in the resistive $g$ turbulence compared with the classical shear flow Kelvin-Helmholtz $(\mathrm{K}-\mathrm{H})$ driven turbulence.

\section{DISCLAIMER}

This report was prepared as an account of work sponsored by an agency of the United States The United States Government nor any agency thereof, nor any of their Government. Nether the Unty, express or implied, or assumes any legal liability or responsiemployees, makes any warmetenes, or usefulness of any information, apparatus, product, or bility for the accuracy, complests that its use would not infringe privately owned rights. Referprocess disclosed, or represents that its use wot, process, or service by trade name, trademark, ence herein to any specific commercial product, process, or manufacturer, or otherwise does not necessarly constiute or inply its encerent The views mendation, or favoring by the United States Government or any agency the hor hecessarily state or reflect those of the and opinions of anthors expressed herein do not
United States Government or any agency thereof. 


\section{Introduction}

J) Jing recent years, many theoretical works on the relation between plasma turbulence and background shear flow have been done $e^{1,2,3,4}, 5,6,7,8,9,10$ in order to understand the physics of $\mathrm{H}$-mode which has been experimentally observed in many tokamaks and searched for in other devices such as stellarators. Reduction of growth rates and saturation levels of many types of instabilities in the presence of background shear flow were theoretically ronfirmed. However drawing a closed picture of the $\mathrm{H}$-mode physics requires a description of the mechanism for the generation of the background flow, and several theoretical models for that have been proposed. These models may be classified into two types. One $\mathrm{i}$; based on neoclassical or particle orbit loss processes ${ }^{2,3}$ and the other based on turbulent processes or Reynolds stress. ${ }^{4,5,6,9,10}$ The latter yields a simpler picture in that a self-consistent treatment of the shear flow generation and its turbulence suppression can be constructed within the framework of the fluid model of the plasma dynamics.

In this work, our main concern is in the shear flow generation by the Reynolds stress in the case of the resistive $g$-modes. ${ }^{11,12,13}$ Here the electrostatic fluid model equations are used for the analytical and numerical calculations in the single-helicity sheared slab configurations. It is known that the eigenfunctions of the pure linear resistive $g$-modes yield no Reynolds stress (the word 'pure' implies the case that neither background flow nor diamagnetic drift effects are taken into account). Therefore, as a seed of background shear flow, we put an external neoclassical flow velocity into the background momertum balance equation. However, significant suppression of the turbulence is not realized by the external shear flow predicted by neoclassical theory acting alon. It will be shown by the quasilinear analysis and the nonlinear simulations that the Reynolds stress generated by the resistive $g$-modes in the presence of the weak neoclassical shear flow enhances the shear flow 
generation mechanism so that the generated shear flow can become much larger than the external neoclassical shear flow, and thus effectively reduce the turbulence level and transport when the collisional flow damping rate is small. This situation is in striking contrast to the case of the Kelvin-Helmholtz (K-H) instability ${ }^{14}, 15$ where the Reynolds stress weakens the background shear flow. This difference is also characterized by the tilting directions ${ }^{14}$ of the vortices in these instabilities, which are opposite to each other. Furthermore, we will find that even in the case that there is no external flow and no diamagnetic drift effect, nonlinear interaction of the pure resistive $g$-modes yields a small seed of background shear flow, which grows up large enough to cause turbulent transport reduction.

This work is organized as follows. In Sec. II, the fluid model equations describing the resistive interchange modes and the background poloidal shear flow are explained. The external shear flow term derived from the neoclassical theory is also described. In Sec. III, we present the quasilinear analysis of the shear flow generation by the Reynolds stress. We find that the eigenfunction of the resistive $g$-mode under the background shear flow yields the Reynolds stress enhancing the flow shear. We also derive the Landau equation for the evolution of the single mode amplitude of the resistive $g$-mode taking account of the shear How generation. In Sec. IV, the shear flow generation and the transport suppression are investigated by the single-helicity nonlinear simulations using the model equations given in Sec. II. Finally, conclusions and discussion are given in Sec. V.

\section{Model Equations}

In order to describe the resistive $g$-mode, we use the following equations ${ }^{12,13}$ for the electrostatic potential $\phi \equiv \phi_{0}+\tilde{\phi}$ and the pressure $p \equiv P_{0}+\tilde{p}$ (the subscript 0 denotes the background part and : the fluctuation part)

$$
\frac{\rho_{m} c}{B_{0}}\left(\frac{\partial}{\partial t}-\mu \nabla_{\perp}^{2}+\frac{c}{B_{0}} \hat{\mathbf{z}} \times \nabla \phi \cdot \nabla\right) \nabla_{\perp}^{2} \phi=-\frac{B_{0}}{c \eta} \nabla_{\|}^{2} \phi-\Omega^{\prime} \frac{\partial \tilde{p}}{\partial y}
$$




$$
\left(\frac{\partial}{\partial t}-\chi \nabla_{\perp}^{2}+\frac{c}{B_{0}} \hat{\mathbf{z}} \times \nabla \phi \cdot \nabla\right) \tilde{p}=\frac{c}{B_{0}} P_{0}^{\prime} \frac{\partial \phi}{\partial y}
$$

where $B_{0}$ is the component of the static magnetic field along the $z$-axis, $\rho_{m}$ the average mass density, $c$ the light velocity in the vacuum, $\eta$ the resistivity, $\mu$ the viscosity, $x$ the pressure diffusivity, $P_{0}^{\prime} \equiv d P_{0} / d x(<0)$ the background pressure gradient and $\Omega^{\prime} \equiv d \Omega / d x(>0)$ the average curvature of the magnetic field line. ${ }^{i 6} \nabla_{\perp}^{2}=\partial_{x}^{2}+\partial_{y}^{2}$ denotes the two-dimensional Laplacian. The gradient along the static sheared magnetic field line is given by

$$
\nabla_{\|}=\frac{\partial}{\partial z}+\frac{x}{L_{3}} \frac{\partial}{\partial y} .
$$

Here $B_{0}, L_{s}, \rho_{m}, \eta, \nu, \chi, P_{0}^{\prime}$ and $\Omega^{\prime}$ are assumed to be constant since we treat a local transport problem. The electrostatic approximation is used in Eqs. (1) and (2) since we consider the low beta plasma in the peripheral region.

The dissipation coefficients $\mu$ and $\chi$ are included to give an energy sink in the high wavenumber region, which is necessary for the turbulence saturation. We consider that they are dominated by the ion-ion collision and given by the classical or neoclassical expressions.

We employ the following equation for the background poloidal flow $v_{E} \equiv\left(c / B_{0}\right)\left(d \phi_{0} / d x\right)$ :

$$
\frac{\partial v^{\prime} E}{\partial t}+\nu\left(v_{E}-V(x)\right)=-\frac{\partial\left\langle\tilde{v}_{x} \tilde{v}_{y}\right)}{\partial x}
$$

Here $\tilde{\mathbf{v}}=\hat{\mathbf{z}} \times \nabla \tilde{\phi}$ and

$$
-\left\langle\tilde{v}_{x} \tilde{v}_{y}\right\rangle
$$

represents the Reynolds stress caused by the potential fluctuations, $\langle\cdot\rangle$ the average in the poloidal $y$-direction, $V$ the external flow and $\nu$ the relaxation rate given by the neoclassical theory as ${ }^{17,18,19}$

$$
\begin{aligned}
& \nu=\frac{\omega_{T} q^{2} \nu_{*}}{1+\nu_{*}} \\
& \omega_{T}=\frac{v_{T i}}{q R}, \quad \nu_{*}=\frac{\nu_{i}}{\omega_{T}(r / R)^{3 / 2}}
\end{aligned}
$$


where $v_{i}$ denotes the ion thermal velocity, $q$ the safety factor, $R$ and $r$ the plasma major and minor radius, respectively.

Hereafter we use the resistive $g$ units given by

$$
\begin{aligned}
& {[t]=\left(-P_{0}^{\prime} \Omega^{\prime} / \rho_{m}\right)^{-1 / 2}=\sqrt{L_{p} L_{c}} / v_{T}} \\
& {[x]=[y]=c L_{s} \eta^{1 / 2}\left(-\rho_{m} P_{0}^{\prime} \Omega^{\prime}\right)^{1 / 4} / B_{0}=L_{s}\left(\nu_{e} v_{T} / \omega_{c e} \omega_{c i} \sqrt{L_{n} L_{c}}\right)^{1 / 2}} \\
& {[z]=L_{s}} \\
& {[\mu]=[\chi]=c[\phi] / B_{0}=[x]^{2} /[t]=c^{2} \eta\left(-P_{0}^{\prime}\right) \Omega^{\prime} L_{s}^{2} / B_{0}^{2}=D_{c l}\left(L_{s}^{2} / L_{p} L_{c}\right)} \\
& {[p]=P_{0}^{\prime}[x]}
\end{aligned}
$$

where $\nu_{e}=n_{e} e^{2} \eta / m_{e}$ is the electron collision frequency, $D_{c l}=c^{2} \eta P_{0} / B_{0}^{2}$ the classical diffusivity. $\omega_{c e}=e B_{0} / m_{e} c, \omega_{c i}=e B_{0} / m_{i} c$ the cyclotron frequencies, $v_{T}=\sqrt{\left(T_{i}+T_{e}\right) / m_{i}}$, $L_{c}=1 / \Omega^{\prime}$ and $L_{p}=-P_{0} / P_{0}^{\prime}$. Then we obtain model equations in non-dimensional variables from Eqs. (1), (2), and (4) as follows:

$$
\begin{aligned}
& \partial_{t} \nabla_{\perp}^{2} \phi+\left[\phi, \nabla_{\perp}^{2} \phi\right]=-\nabla_{\|}^{2} \phi-\partial_{y} \tilde{p}+\mu \nabla_{\perp}^{4} \phi \\
& \partial_{t} \tilde{p}+[\phi, \tilde{p}]=-\partial_{y} \phi+\chi \nabla_{\perp}^{2} \tilde{p} \\
& \partial_{t} v_{E}+\nu\left(v_{E}-V\right)=-\partial_{x}\left\langle\tilde{v}_{x} \tilde{v}_{y}\right\rangle
\end{aligned}
$$

where

$$
\begin{aligned}
& v_{E} \equiv d \phi_{0} / d x, \\
& \nabla_{\|}=\partial_{z}+x \partial_{y},
\end{aligned}
$$

and

$$
[f, g]=\left(\partial_{x} f\right)\left(\partial_{y} g\right)-\left(\partial_{x} g\right)\left(\partial_{y} f\right)
$$




\section{Quasilinear Analysis}

We now consider a linear mode of the form $\tilde{\phi}=\phi_{k}(x) e^{i k y-i \omega t}$ under the background shear flow $v_{E}(x)$, with the mode resonant surface of which is located at $x=0$. Linearization of Eqs. (1) and (2) yields the following mode equation

$$
[\frac{d^{2}}{d x^{2}}-k^{2}+\underbrace{\frac{k v_{E}^{\prime \prime}}{\omega-k v_{E}}}_{\mathrm{K}-\mathrm{H}}-\underbrace{\frac{k^{2}}{\left(\omega-k v_{E}\right)^{2}}}_{g \text {-mode }}-\underbrace{\frac{i k^{2} x^{2}}{\omega-k v_{E}}}_{\text {magnetic shear }}] \phi_{k}(x)=0
$$

where the viscosity $\mu$ and pressure diffusivity $\chi$ were neglected and the last three terms in the left-hand side corresponds to Kelvin-Helmholtz (K-H),g-mode, and magnetic shear effects, respectively. Contribution from the mode $\phi_{k}(x)=\left|\phi_{k}(x)\right| \exp \left[i \theta_{k}(x)\right]$ to the poloidal acceleration due to quasilinear Reynolds stress is given by

$$
\begin{aligned}
-\frac{\partial\left\langle\tilde{v}_{x} \tilde{v}_{y}\right\rangle}{\partial x} & =i k \frac{d}{d x}\left(\phi_{k} \frac{d \phi_{k}^{*}}{d x}-\phi_{k}^{*} \frac{d \phi_{k}}{d x}\right)=2 k \frac{d}{d x}\left(\left|\phi_{k}\right|^{2} \frac{d \theta_{k}}{d x}\right) \\
& =2\left|\phi_{k}\right|^{2}\left[\omega_{i}\{\underbrace{\frac{k^{2} v_{E}^{\prime \prime}}{\left|\omega-k v_{E}\right|^{2}}}_{\mathrm{K}-\mathrm{H}}+\underbrace{\frac{2 k^{4} v_{E}}{\left|\omega-k v_{E}\right|^{4}}\left(1-\frac{\omega_{\tau}}{k v_{E}}\right)}_{g \text {-mode }}\}\right. \\
& -\underbrace{\frac{k^{4} x^{2} v_{E}}{\left|\omega-k v_{E}\right|^{2}}\left(1-\frac{\omega_{\tau}}{k v_{E}}\right)}_{\text {magnetic shear }}]
\end{aligned}
$$

where we used Eq. (13) and $\omega=\omega_{r}+i \omega_{i}$. If we assume that the background poloidal shear flow $v_{E}(x)$ has the profile in the form of $v_{E}(x)=v_{0} \tanh \left(x / L_{E}\right)$, or $v_{E}(x)=v_{0} \sin \left(x / L_{E}\right)$, we find that the sign of $v_{E}^{\prime \prime} \equiv d^{2} v_{E} / d x^{2}$ is opposite to that of $v_{E}$ and that we can put $\omega_{r}=0$ and $\theta_{k}(-x)=-\theta_{k}(x)$. Then it can be seen from Eq. (14) that, for the unstable mode $\omega_{i}>0$, the $\mathrm{K} \cdot \mathrm{H}$ and magnetic shear terms weaken the shear flow while the $g$-mode term strengthens it. 
Characterizing the general shear flow profile $v_{E}(x)$ in terms of its maximum value $v_{0}$ and charcteristic length $L_{E}$, the ratio of the $g$-mode term to the K-H term in Eq.(14) is estimated as $2 k^{2}\left(v_{E} / v_{E}^{\prime \prime}\right)\left(\gamma^{2}+k^{2} v_{E}^{2}\right)^{-1} \simeq(2 G /(1+G))\left(L_{E} / \Delta\right)^{2}$ where $\Delta$ denotes the radial mode width and $G \equiv\left(L_{E} / v_{0}\right)^{2}\left(v_{T}^{2} / L_{p} L_{c}\right)$. In making this estimate, we use that for low wavenumbers the resistive $g$ growth rate $\gamma$ and mode width $\Delta$ are related by $\gamma / k^{2} \Delta \sim 1$ in our units (8). From Ref. $\neg, G>1$ is required for the unstable resistive $g$-mode. Considering the typical case where $\lrcorner<L_{E}$, we find that the contribution of the $g$-mode term to the shear flow generation overcomes the damping due to $\mathrm{K}$ - $\mathrm{H}$ term in the $g$-mode unstable case. As the shear flow grows. the magnitude of $G$ decreases. Then the relative contribution from the $g$-mode term also decreases and the fluctuations are more stabilized so that the Reynolds stress effect is weakened. Thus the shear flow generation by the Reynolds stress is considered to work more effectively in the L-H transition phase than in the $\mathrm{H}$-mode phase. When the shear flow becomes extremely large, the K-H term is the dominant ontribution and the $\mathrm{K} \cdot \mathrm{H}$ instability appears instead of the resistive $\mathrm{g}$-mode. However, the criterion for the K-H instability $y^{i, 13}$ is not satisfied in the practical experimental parameters and this K-H unstable case is not considered in the simulations in the next section.

For simplicity, let us consider the potential eigenfunction of the form

$$
\phi_{k}(x)=\left|\phi_{k}(x)\right| \exp (i k \alpha x)
$$

where $\alpha$ represents a tilting angle of the vortex with respect to the poloidal direction. In this case, we have

$$
-\frac{\partial\left\langle\tilde{v}_{x} \tilde{v}_{y}\right\rangle}{\partial x}=2 k^{2} \alpha \frac{d\left|\phi_{k}(x)\right|^{2}}{d x}
$$

Figures $1(a)$ and (b) show the contours of the potential eigenfunctions given by Eq. (15) with different signs of tilting angles $\alpha$. Since generally $x d\left|\phi_{k}(x)\right|^{2} / d x$ is negative, Eq. (16) shows that the background shear flow is decreased by the Reynolds stress for $\alpha v_{E}^{\prime}>0$ [Fig. 1(a)] but increased for $\alpha v_{E}^{\prime}<0$ [Fig. 1(b)]. For the pure K-H instability, it was confirmed in 
Ref. 14 that the vortex tilting satisfied the condition $\alpha v_{E}^{\prime}>0$ and weakened the shear flow, which agrees with the our present prediction based on Eq. (14). On the other hand, for the resistive $g$-mode under the background shear flow, we will verify later by simulations that the direction of the vortex tilting is opposite to that of the pure $\mathrm{K} \cdot \mathrm{H}$ instability and the shear flow is enhanced by the Reynolds stress.

Next, we treat the resistive $g$-mode under the background poloidal shear flow near the marginally stable state by the weakly nonlinear theory. ${ }^{20,21,22}$ Here, we assume that the system is weakly unstable and that the maximum linear growth rate is slightly larger than zero. In such a weakly unstable state, fluctuation amplitudes and their temporal variation are small, and their smallness are represented by the parameter $\lambda$. We make the following perturbation expansion with the parameter $\lambda$ :

$$
\begin{aligned}
\left(\begin{array}{c}
\tilde{\phi} \\
\tilde{p}
\end{array}\right) & =\lambda\left(\begin{array}{l}
\tilde{\phi}_{1} \\
\tilde{p}_{1}
\end{array}\right)+\lambda^{2}\left(\begin{array}{l}
\tilde{\phi}_{2} \\
\tilde{p}_{2}
\end{array}\right)+\cdots \cdot \\
\frac{\partial}{\partial t} & =\lambda \frac{\partial}{\partial \tau_{1}}+\lambda^{2} \frac{\partial}{\partial \tau_{2}}+\cdots \cdot \\
v_{E} & =v_{E 0}+\lambda v_{E 1}+\lambda^{2} v_{E 2}+\cdots \cdots .
\end{aligned}
$$

Since $\lambda$ is only the formal expansion parameter, we can put $\lambda=1$ in the final results or, in other words, $\lambda$ can be included into the fluctuation amplitudes and time derivatives themselves. We put the external poloidal flow $V(x)$ as

$$
V=V_{c}+\lambda^{2} V_{2}
$$

In the marginally stable state $\lambda=0$, Eqs. (11) and (18) give the background poloidal flow $v_{E}=V_{c}$, for which the maximum growth rate of the mode with the lowest wavenumber $k$ is zero. It should be noted that the marginal stability requires finite values for $\mu$ and $\chi$. For $\lambda \neq 0$, the system becomes linearly unstable since we assume that $V_{2}$ in the external flow weakens the shear of $V_{c}$ and lowers its stabilizing effect. 
In $O(\lambda)$, Eqs. (9) and (10) yield the linear eigenmode equations, the solntion of which is written as

$$
\left(\begin{array}{c}
\tilde{\phi}_{1} \\
\tilde{p}_{1}
\end{array}\right)=A e^{i k y}\left(\begin{array}{c}
\phi_{1}(x) \\
p_{1}(x)
\end{array}\right)+A^{*} e^{-i k y}\left(\begin{array}{c}
\phi_{1}^{*}(x) \\
p_{1}^{*}(x)
\end{array}\right)
$$

where we considered the mode with the resonant surface at $x=0$ only. Here $\phi_{1}(x)$ and $p_{1}(x)$ are the eigenfunctions obtained from the $O(\lambda)$ equations although we need to proceed to higher order equations to determine the amplitude $A$.

In the $O\left(\lambda^{2}\right)$ equations, the beating of the first-order fluctuations $\tilde{\phi}_{1}$ and $\tilde{p}_{1}$ produces the second-order fluctuations which have the poloidal wavenumbers 0 and $2 k$. From the solvability condition of the $O\left(\lambda^{2}\right)$ equations for the wavenumber $k$, we have

$$
\partial_{\tau_{1}} A=0
$$

Hereafter, in the $O\left(\lambda^{2}\right)$ perturbations, we neglect the effects of the fluctuations with the poloidal wavenumber $2 k$ and take account of only the background pressure and poloidal flow modifications which correspond to the poloidal wavenumber 0 . These background corrections are the dominant $O\left(\lambda^{2}\right)$ effects in the cases presented here and are derived from Eqs. (10) and (11) as

$$
\begin{aligned}
p_{2}^{\prime} & =\frac{2 k}{\chi}|A|^{2} \operatorname{Imag}\left(p_{1}^{*} \phi_{1}\right) \equiv|A|^{2} p_{Q L}^{\prime} \\
v_{E 2} & =V_{2}+\frac{2 k}{\nu}|A|^{2} \operatorname{Imag}\left[\partial_{x}\left(\phi_{1}^{*} \partial_{x} \phi_{1}\right)\right] \equiv V_{2}+|A|^{2} v_{Q L}
\end{aligned}
$$

where $|A|^{2} p_{Q L}^{\prime}$ denotes tine quasilinear correction for the background pressure gradient, $V_{2}$ the deviation of the external flow from its critical profile which gives the linear instability, and $|A|^{2} v_{Q L}$ represents the background poloidal flow generated nonlinearly (or quasilinearly) by the Reynolds stress due to the first-order potential fluctuations.

We now obtain the following Landau equation from the solvability condition of the $D\left(\lambda^{3}\right)$ equations from the secular variation at the wavenumber $k$ :

$$
\partial_{t} A=\left(-i \omega-D|A|^{2}\right) A
$$


Here $\omega=\omega_{r}+i \omega_{i}$ is the linear eigenfrequency and $D=D_{r}+i D_{i}$ is the complex valued constant determined by the shape and phase of the eigenmode. The third order solvability condition gives the formulas

$$
\begin{aligned}
-i \omega & =D_{1} / D_{0} \\
D & =D_{3} / D_{0}
\end{aligned}
$$

where

$$
\begin{aligned}
& D_{0}=\int d x\left[\phi_{1}^{\dagger}\left(-\partial_{x}^{2}+k^{2}\right) \phi_{1}-p_{1}^{\dagger} p_{1}\right] \\
& D_{1}=-i k \int d x\left[V_{2}^{\prime}\left\{\phi_{1}^{\dagger}\left(-\partial_{x}^{2}+k^{2}\right) \phi_{1}-p_{1}^{\dagger} p_{1}\right\}+V_{2}^{\prime \prime} \phi_{1}^{\dagger} \phi_{1}\right] . \\
& D_{3}=i k \int d x\left[v^{\prime} Q L\left\{\phi_{1}^{\dagger}\left(-\partial_{x}^{2}+k^{2}\right) \phi_{1}-p_{1}^{\dagger} p_{1}\right\}+v_{Q L}^{\prime \prime} \phi_{1}^{\dagger} \phi_{1}+p_{Q L}^{\prime} p_{1}^{\dagger} \phi_{1}\right]
\end{aligned}
$$

where $\phi_{i}^{\dagger}$ and $p_{1}^{\dagger}$ are the eigenfunctions determined by the linear equations adjoint to those for $\rho_{1}$ and $p_{1}$. The Landau equation $(23)$ is analytically solved for the growth and saturation of the single mode amplitude given by

$$
|A(t)|^{2}=\frac{|A(0)|^{2}}{\left(D_{r} / 2 \omega_{i}\right)|A(0)|^{2}+\left[1-\left(D_{r} / 2 \omega_{1}\right)|A(0)|^{2}\right] \exp \left(-2 \omega_{i} t\right)} .
$$

Generally the quasilinear pressure flattening represented by $p_{Q L}^{\prime}$ contributes to the saturation of the amplitude and we will see later that the flow generated by the Reynolds stress $v_{Q L}$ also causes the amplitude saturation in both cases of the pure $\mathrm{K}-\mathrm{H}$ instability and the resistive $g$-mode under the background poloidal shear flow. The saturation amplitude and the convective transport are given by

$$
\begin{aligned}
& |A|^{2}=\frac{2 \omega_{i}}{D_{r}} \\
& \left\langle\tilde{p} \tilde{c}_{r}\right\rangle=2 k|A|^{2} \operatorname{Imag}\left(p_{1}^{*} \phi_{1}\right) .
\end{aligned}
$$

If we assume that $v_{E}(-x)=-v_{E}(x)$, we have a pure imaginary linear eigenfrequency $\omega^{\prime}=$ $L_{i}$, and a complex-valued eigenfunction $\phi_{1}(x)$ satisfying $\left|\phi_{1}(-x)\right|=\left|\phi_{1}(x)\right|$ and arg $\phi_{1}(-x)=$ 
$-\arg \phi_{1}(x)$. Then $D_{0}, D_{1}$ and $D_{3}$ are all real-valued constants. For linear instability, the deviation $V_{2}$ of the external poloidal $A_{\text {sw }}$ fiom that in the marginal stable state is required to increase the shear for the pure $\mathrm{K} \cdot \mathrm{H}$ instability case and decrease it for the resistive $g$-mode case. As we found previously in this section, the Reynolds-stress-produced flow v'QL works opposite to $V_{2}$. Then, comparing the contribution of $V_{2}$ to $D_{1}$ with that of $v_{Q L}$ to $D_{3}$ in Eq. (24), we see that $D_{1}$ and $D_{3}$ (or $\omega_{i}$ and $D$ ) have the same sign. Thus $D$ is positive and $v^{\prime} Q L$ contributes to the amplitude saturation in both the K-H and the resistive $g$-mode cases. From Eqs. (22) and (24), $\iota^{\prime} Q L$ and $D_{3}$ are inversely proportional to $\nu$ so that the saturation amplitude (26) and the transport flux (27) are lowered for smaller values of $\nu$.

Analytical treatments in this section are quantitavely accurate in the system near the marginally stable state while they give only qualitative suggestions for the behavior in the strongly nonlinear or turbulent state, in which it is difficult to apply analytical methods and numerical solution as in Sec. IV is required. Here we emphasize the analytically predicted tendencies that, under the background shear flow, the Reynolds stress due to the resistive $g$ mode enhances the shear in contrast with the $\mathrm{K}-\mathrm{H}$ case and that larger shear flow generation and smaller saturated transport are obtained for smaller values of $\nu$.

\section{Simulation Results}

Equations $(9)-(11)$ are numerically solved in the sheared slab configuration. Here we concentrate on the single-helicity (or two-dimensional) nonlinear simulations, in which all the the Fourier modes of fluctuations are assumed to have the same mode resonant surface at $x=0$. We put the boundaries at $x= \pm a$, which are chosen such that the fluctuations are damped enough by the magnetic shear before reaching the boundaries. We use the external poloidal flow profile in the form of

$$
V(x)=V_{0} \sin \left(\frac{\pi x}{2 a}\right)
$$


which gives the vorticity as

$$
V^{\prime}(x)=V_{0}^{\prime} \cos (\pi x / 2 a)
$$

where $V_{0}^{\prime}=\pi V_{0} / 2 a$ gives the maximum vorticity (or shear). We employ the boundary conditions given by

$$
\tilde{\phi}=\partial_{x}^{2} \tilde{\phi}=\tilde{p}=0 \quad \text { at } \quad x= \pm a
$$

As initial conditions of the simulations, we give perturbations to all the single-helicity modes in the form of the linear eigenfunctions with the same kinetic energy and the random phase relation to each other. Since our concerns in this study are with the background shear flow production due to the Reynolds stress, we neglect the quasilinear pressure flattening and fix the background pressure gradient, which is equivalent to the addition of a certain source term to the pressure equation. In all the simulations here, the normalized viscosity and pressure diffusivity are given by $\mu=\chi=4.0$. In the dimensional form, they are written as $\mu=\gamma=4.0 D_{c l} L_{\mathbf{s}}^{2} / L_{p} L_{c}$ which corresponds to the order of the ion classical diffusivity since typically we have $L_{s}^{2} / L_{p} L_{c} \sim 10$. (When we employ the classical ion viscosity and thermal diffusivity, the non-dimensional parameters $\mu$ and $\chi$ normalized by $[x]^{2} /[t]=D_{c l} L_{s}^{2} / L_{p} L_{c}$ are independent of plasma density and temperature but only depending on the ratio $T_{e} / T_{i}$ and the geometrical factor $L_{s}^{2} / L_{p} L_{c}$.) The potential and pressure fluctuations are Fourierexpanded as

$$
\left(\begin{array}{c}
\tilde{\phi} \\
\tilde{p}
\end{array}\right)=\sum_{m}\left(\begin{array}{l}
\phi_{m}(x, t) \\
p_{m}(x, t)
\end{array}\right) \exp (i m k y)
$$

where $k \equiv 2 \pi / L_{y}$ is the minimum poloidal wavenumber and we use $k=1 / 40$ in the simulations. From Eq. (8), the unit length $[y]$ depends on the plasma density and temperature etc. and typically $[y] / \rho_{i} \simeq 0.1-1.0$ ( $\rho_{i}$ : the ion thermal Larmor radius). Then the maximum poloidal wavelength $L_{y}$ used here corresponds to 10-100 times larger than $\rho_{i}$.

Figure 2 shows the linear growth rates for the resistive $g$-mode under the background poloidal flow given by the external one [Eq. (28)]. When the background poloidal flow has 
the maximum shear $V_{0}^{\prime}=0.2$, the system is completely linear stable.

The nonlinear time evolution of the kinetic energy and the convective transport are shown in Figs. 3 and 4. There the same external shear flow with $V_{0}^{\prime}=0.1$ is used and the same initial perturbation is given to the $m=1$ rode potential. The only difference in the parameters for Figs. 3 and 4 is the values of the relaxation rate $\nu$ in the momentum balance Eq. (11): $\nu=0.02$ in Fig. 3 and $\nu=0.3$ in Fig. 4 . In both figures, we can see that the $m=1$ mode, which is the most unstable mode with the minimum poloidal wavenumber, gives the dominant contribution to the kinetic energy and the transport in the nonlinearly saturated states. The most remarkable feature of the comparison is that, in the sma"! $\nu$ case of Fig. 3, a strong background $(m=0)$ poloidal flow is generated and that this flow strongly reduces the fluctuation kinetic energy and the associated radial thermal transport. On the other hand, in the large $\nu$ case of Fig. 4, neither generation of a significant background flow, nor the reduction of the fluctuation kinetic energy and the transport occur. These results are qualitatively consistent with those of the quasilinear analysis in the previous section, where it was shown that the Reynolds-stress-produced flow increases and the saturation amplitude decreases as $\nu$ becomes smaller. The temporal evolution described by Eq. (25) qualitatively agree with the numerical results in Figs. 3 and 4 in that Eq. (25) shows the saturation of the dominant $m=1$ mode as well as its linear growth although it fails to describe the viershooting observed in Fig. 3, which may be due to highly nonlinear effects.

Figure 5 shows radial profiles of the convective flux $\left\langle\tilde{p} \tilde{v}_{x}\right\rangle$ in the cases of Figs. 3 and 4 . Both profiles are of the Gaussian form with the same radial width. The radial structures of the $m=1$ mode potential and pressure in these cases are almost the same as those of the linear eigenfunctions. The flux amplitude for $\nu=0.02$ is about $3 / 4$ of that for $\nu=0.3$.

Figure 6 shows radial profiles of the background poloidal flow $v_{E}(x)$ and the vorticity $v_{E}^{\prime}(x)$ at the time $t=2000$ in the case of Fig. 3. There profiles of the external flow $V(x)$ and vorticity $V^{\prime}(x)$ are also plotted by the dashed lines. It is seen that the maximum shear (or 
vorticity) of the background flow generated by the Reynolds stress is about 6 times larger than that of the external flow. At this shear of the background flow, linear perturbation theory gives the completely stabilized state with no fluctuations. However, the saturated or quasi-steady state with finite amplitude of fluctuations is obtained in the simulation of Fig. 3.

The contours of the $m=1$ mode of the electrostatic potential in the case of Fig. 3 are shown in Fig. 7 . There (a) is for the growing state $(t=500)$ and $(b)$ for the saturated state $(t=2000)$. It is found that the vortex tilts in the same direction as in Fig. 1(b), which implies the Reynolds stress enhancing the shear of the background poloidal flow. The vortex tilting is clearer in (a) than in (b). In the case (a) the shear flow generation due to the Reynolds stress exceeds the collisional damping (denoted by $\nu$ ) while in the case (b) they are balanced with each other. In the saturated state (b), the fluctuation amplitude is high and therefore only a small tilting angle is required to maintain the shear flow if $\nu$ is small.

Figure 8 shows the convective flux $\left\langle\widetilde{p}_{x}\right\rangle$ and the background poloidal flow shear $v_{E}^{\prime}$ at the mode resonant surface $x=0$ for $\nu=0.02$ and $\nu=0.3$. The solid circles correspond to the case of $V_{0}^{\prime}=0.05$ and the open circles to the case of $V_{0}^{\prime}=0.1$. Also plotted is the convective flux in the case where the background flow is fixed to be zero. For smaller $\nu$, the Reynolds stress generates a higher shear in the background shear flow and thus a larger reduction of the transport. In the cases of no background flow ard $V_{0}^{\prime}=0.05$, the dominant poloidal mode number in the saturated state is $m=2$, which corresponds to the most linearly unstable mode. On the other hand, for $V_{0}^{\prime}=0.1$, the $m=1$ mode, which has the maximum linear growth rate, becomes dominant in the saturated state and gives larger transport than in the $m=2$ mode dominant case. In the saturated states, we always found that only one mode is dominant and contributions from the other modes to the energy and transport are much smaller than the dominant mode contribution. (We never observed the cases in which both the $m=1$ and $m=2$ modes had comparable amplitudes.) It seems that the mode which 
has grown faster suppresses the other modes.

In Fig. 9, the convective flux, the background poloidal flow shear at $x=0$ and the maximum linear growth rate are shown for different values of $V_{0}^{\prime}$. Here $\nu=0.02$ is used. The dominant poloidal mode number in the saturated state is $m=2$ for $V_{0}^{\prime}=0,0.02,0.05$ and $m=1$ for $V_{0}^{\prime}=0.1$. In the $m=2$ mode dominant cases, the convective flux gradually decreases as $V_{0}^{\prime}$ increases. However, in the $m=1$ mode dominant case, larger transport is found again in spite of the smaller linear growth rate and the larger Reynolds-stressproduced background vorticity. Generally, nonlinear saturation levels of modes with smaller wavenumbers tend to be less affected by the reduction of the linear growth rates and give larger transport.

As seen from Fig. 3, any fluctuation with small amplitude damps in the course of time for $V_{0}^{\prime} \geq 0.2$. Figures $10-12$ shows the time evolution of the kinetic energy and the turbulent transport for two cases in which the external parameters are changed halfway through the time interval. In Figs. 10 and 11, we put $v_{E}=0$ for $0<t<1000$, and then subsequently $v_{E}$ is given by solving Eq. (11) with $V_{0}^{\prime}=0.4$ for $t>1000$. Here $\nu=0.3$ is used in Fig. 10 and $\nu=0.02$ in Fig. 11. In the large $\nu$ case (Fig. 10), the background flow $v_{E}$ approaches to the external one $V_{0}$ immediately after $t=1000$. Then the fluctuation energy decreases and the transport is reduced to less than $1 / 7$ of that in the no shear flow case $(t<1000)$. We should note that the value of the maximum background vorticity $V_{0}^{\prime}=0.4$, causing the significant reduction of the turbulent transport, is about twice that required for the linear marginal stability of the system (see Fig. 2) although the transport is not reduced in the external flow with $V_{0}^{\prime}=0.1$ (see the case of $V_{0}^{\prime}=0.1$ and $\nu=0.3$ in Fig. 8) for which the linear growth rates is considerably decreased. (We already found the large background vorticity $\simeq 0.7$ in Fig. 6, where the decrease of the transport was also observed.) Thus, in the nonlinear turbulent regime, significant transport reduction can be realized only when the background flow has a shear that is much higher than that predicted by the linear stability criterion. In 
the small $\nu$ case (Fig. 11), the Reynolds stress enables the background shear flow to exceed the external flow after $t=1000$, which leads to the complete suppression of the fluctuations and no turbulent transport.

In Fig. 12, no external flow $\left(V_{0}=0.0\right)$ is given for $t>1000$ while $\nu$ is the same as in Fig. $11(\nu=0.02)$. In this case, we also find that the Reynolds stress effectively produces the back ground flow, which reduces the fluctuation level and the transport. The shear flow generation and the transport reduction occur at $t \sim 1200$ with some delay after the change of the simulation conditions. The vortex tilting required for the non-vanishing Reynolds stress in this case is produced not by the external shear flow $V_{0}$ but by the nonlinear interactions of the modes with different wavenumbers. This interaction process will be discussed in the next section. Interestingly, a small decrease in the background shear flow accompanied with an increase of the fluctuation and the transport is observed suddenly at $t \sim 1900$, when no external change of the plasma conditions (like that at $t=1000$ ) is introduced.

Up to this point, we fixed the values of $\mu, \chi$ and $k=2 \pi / L_{y}$. The numerical results can be influenced by these dissipation coefficients as well as the maximum poloidal wavelength $L_{y}$. These parameters determine the number of the modes which need to be included in the simulations for obtaining the nonlinearly saturated states. Simulations using different values for these parameters were also done and we found that, for larger $L_{y}$ with fixed $\mu$ and $\chi$ (or for smaller $\mu$ and $\chi$ with fixed $L_{y}$ ), behavior of fluctuations becomes more turbulent and the high-wavenumber Reynolds stress contribution to the flow damping increases in the steady turbulence phase although the shear flow generation by the Reynolds stress still appears in the transition phase.

We consider that the change in $\nu$ as seen in Figs. 10-12 may be triggered by the auxiliary heating which decreases collisionality. Since $\nu$ can change also due to the change in the background plasma parameters, which is disregarded in the analysis in Sec. III, a self-consistent treatment of mixed mechanisms of the background response, change in $\nu$, fluctuations and 
shear flow is required for realistic description of the $\mathrm{L}-\mathrm{H}$ transition and remains for a future problem.

\section{Conclusions and Discussion}

In this work, we investigate the generation of shear flow by the Reynolds stress arising from self-consistent pressure gradient driven fluctuations and the resultant reduction of the turbulent transport in the case of the resistive g-modes. As found in Eqs. (14) or (16), the radial variation of the phase of the fluctuation, or the vortex tilting is required to yield the nonvanishing Reynolds stress. Such phase variation is not obtained for the pure linear resistive $g$-modes in the case of no background shear flow, but it is found in the modes under the effect of a small background shear flow as seen in Sec. 1II, and the phase variation is also produced by the nonlinear mode interactions as confirmed through the shear flow generation in Fig. 12. Shear flow effects were also investigated by Carreras et al. ${ }^{4,9,10}$ in the cases where the shear flow is given initially or by diamagnetic effects. The shear flow generation due to the nonlinear interaction of the resistive $g$-modes in the case of no external and no diamagnetic flow and the important role of the collisional flow damping parameter $\nu$ in the L-H transition were first pointed out in our work. The production of the phase variation by the nonlinear interaction is described as follows. For example, let us consider linear modes $m=2.3,4$ which have constant but different phases. Such a situation is possible even in the pure $g$-mode case. Nonlinear interactions of the $m=2$ and $m=3$ modes and that of the $m=3$ and $m=4$ modes both contribute to the production of the $m=1$ mode. If we represent the contributions of these nonlinear interactions to the $m=1$ mode by $\emptyset_{a}(x)=\left|\phi_{a}(x)\right| e^{i \theta_{a}}$ and $\phi_{b}(x)=\left|\phi_{b}(x)\right| e^{i \theta_{b}}$, the phases $\theta_{a}$ and $\theta_{b}$ are constants. However. the sum of them $\left|\phi_{a}(x)\right| e^{i \theta_{a}}+\left|\phi_{b}(x)\right| e^{i \theta_{b}}=|| \phi_{a}(x)\left|e^{i \theta_{a}}+\right| \phi_{b}(x)\left|e^{i \theta_{b}}\right| e^{i \theta(x)}$ has a $x$-dependent phase $\theta(x)$ since $\left|\phi_{a}(x)\right|$ and $\left|\phi_{b}(x)\right|$ generally have different profiles. Thus the nonlinearly produced $m=1$ mode has a radially varying phase and yields the nonvanishing Reynolds 
stress, which, in turn, causes the background shear flow.

Once the background shear flow is generated, the radial dependence of the mode phase appears even through the linear process. In Sec. III, we found that the resistive $g$-mode in the background shear flow has a phase variation or vortex tilting that enhances the shear flow, which is in sharp contrast to the pure K-H instability case. In the $\mathrm{K}-\mathrm{H}$ case, the shear flow is weakened by the vortex tilting. However, as found in Sec. III and Sec. IV, both the $\mathrm{K}-\mathrm{H}$ instability and the resistive $g$-mode arranges the shear flow such that their growth rates are reduced and therefore the system approaches to the stationary state. It is instructive to consider these mechanisms from the point of view based on the energetics. Neglecting all the dissipation terms in Eqs. (9)-(11), we obtain the following energy conservation law

$$
\frac{d}{d t} \int_{-a}^{+a} d x\left(\frac{1}{2} v_{E}^{2}+\frac{1}{2}\left\langle\bar{v}^{2}\right\rangle-x P_{0}\right)=0
$$

where the contributions from the boundary surfaces are assumed to vanish. Here the first term in the left-hand side is the kinetic energy of the background flow, the second term is the turbulent kinetic energy and the last term is the released plasma internal er.ergy. In the reference frame where the volume-averaged flow velocity $\frac{1}{2 a} \int_{-a}^{+a} d x v_{E}(x)$ vanishes, as seen in Sec. IV, the background kinetic energy $\frac{1}{2} \int d x v_{E}^{2}$ is also a measure of the flow shear or the vorticity. The transfer rates between the three energy components of energy balance equation are given by

$$
\begin{aligned}
\frac{d}{d t} \int d x \frac{1}{2} v_{E}^{2} & =-\int d x v_{E} \frac{\partial\left\langle\tilde{v}_{x} \tilde{v}_{y}\right\rangle}{\partial x} \\
\frac{d}{d t} \int d x \frac{1}{2} \tilde{v}^{2} & =\int d x\left(v_{E} \frac{\partial\left\langle\tilde{v}_{x} \tilde{v}_{y}\right\rangle}{\partial x}+\left\langle\tilde{p} \tilde{v}_{x}\right\rangle\right) \\
\frac{d}{d t} \int d x\left(-x P_{0}\right) & =-\int d x\left\langle\tilde{p}_{x}\right\rangle .
\end{aligned}
$$

In the pure $\mathrm{K}-\mathrm{H}$ instability case where the pressure gradient terms are not taken into account. the turbulent kinetic energy is supplied by the background shear flow in the growing phase 
of the instability and therefore we find from the above equations that $-\partial\left\langle\tilde{v}_{x} \tilde{v}_{y}\right\rangle / \partial x$ has the opposite sign of $v_{E}$, i.e., the Reynolds stress reduces the background shear flow at the rate given by Eq. (33). On the other hand, in the resistive $g$-mode case, the turbulent energy comes from the plasma internal energy (or the background pressure gradient) in the form of the flux $\left\langle\tilde{p} \tilde{v}_{x}\right\rangle$. In this case, the background shear flow weakens the growth of the mode so that the term $\int d x v_{E}\left(\partial\left\langle\tilde{v}_{x} \tilde{v}_{y}\right\rangle / \partial x\right)=-\int d x\left\langle\tilde{v}_{x} \tilde{v}_{y}\right\rangle\left(\partial v_{E} / \partial x\right)$ is negative, which implies that $-\partial\left\langle\tilde{v}_{x} \tilde{v}_{y}\right\rangle / \partial x$ has the same sign as $v_{E}$, i.e., the Reynolds stress enhances the background shear flow.

In conclusion we have shown in some detail how the Reynolds stress driven by the selfconsistent plasma fluctuations works effectively for the generation of a shear flow that reduces the thermal transport when the collisional relaxation parameter $\nu$ for the poloidal flow velocity is small which corresponds to ion temperatures above a critical value. The selfgenerated stabilization of the fluctuation driven transport is especially abrupt and strong. Thus the coupled turbulent transport and poloidal flow model presented here for the onset of a reduced transport regime gives a possible paradigm for the experimentally observed rapid L-H transition. The experimental correlation of the transport reduction based on this Reynolds stress mechanism remains an interesting problem. In this work, only the singlehelicity resistive $g$-mode case is examined. As a future task, we need to investigate the shear flow generation by the Reynolds stress in the case of multihelicity turbulence and other types of modes such as the ion pressure gradient driven drift wave turbulence.

\section{Acknowledgments}

The authors acknowledge useful discussions with P.N. Yushmanov, J.Q. Dong, X.N. Su, and M. Wakatani. One of the authors (HS) thanks M. Okamoto for his encouragements of this work. Most of the work was done during the visit of HS at the Institute for Fusion Studies as an exchange scientist of the Joint Institute for Fusion Theory. The numerical simulation 
was done on the computer at the National Institute for Fusion Science.

The work was partially supported by the U.S. Department of Energy grant \#DE-FG0580ET-53088. 


\section{References}

${ }^{1}$ H. Biglari, P.H. Di mond, and P.W. Terry, Phys. Fluids B 2, 1 (1990).

${ }^{2}$ K.C. Shaing and E.C. Crume, Jr., Phys. Rev. Lett. 63, 2369 (1989).

${ }^{3}$ S.-I. Itoh and K. Itoh, Phys. Rev. Lett. 60, 2276 (1988).

${ }^{4}$ B.A. Carreras and V.E. Lynch, Phys. Fluids B 3, 1438 (1991).

${ }^{5}$ P.H. Diamond and Y.-B. Kim, Phys. Fluids B 3, 1626 (1991).

${ }^{6}$ J.F. Drake, J.M. Finn, P. Guzdar, V. Shapiro, V. Shevchenko, F. Waelbroeck, A.B. Hassam. C.S. Liu, and R. Sagdeev, Phys. Fluids B 4, 488 (1992).

'H. Sugama and .I. Wakatani, Phys. Fluids B 3, 1110 (1991).

${ }^{8}$ S. Hamaguchi and W. Horton, Phys. Fluids B 4, 319 (1992).

${ }^{9}$ B.A. Carreras, V.E. Lynch, L. Garcia, and P.H. Diamond, Phys. Fluids B 5, 1491 (1993).

${ }^{10}$ B.A. Carreras, V.E. Lynch, and L. Garcia, Phys. Fluids B 5, 1795 (1993).

${ }^{11}$ H. P. Furth. J. Killeen, and M. N. Rosenbluth, Phys. Fluids 6, 459 (1963).

${ }^{12}$ B.A. Carreras, L. Garcia, and P.H. Diamond, Phys. Fluids 30, 1388 (1987).

${ }^{13}$ H. Sugama and M. Wakatani, J. Phys. Soc. Jpn. 57, 2010 (1988).

${ }^{14}$ W. Horton, T. Tajima, and T. Kamimura, Phys. Fluids 30, 3485 (1987).

${ }^{15}$ T. Chiueh, P.W. Terry, P.H. Diamond, and J.E. Sedlak, Phys. Fluids 29, 231 (1986).

${ }^{16}$ H.R. Strauss, Plasma Phys. 22, 733 (1980). 
${ }^{17}$ W. Horton, X. Su, P.N. Yushmanov, and J. Dong, IFS preprint (1993).

${ }^{18}$ A.B. Hassam and R.M. Kulsrud, Phys. Fluids 21, 2271 (1978).

${ }^{19}$ S.P. Hirshman and D.J. Sigmar, Phys. Fluids 20, 518 (1977).

${ }^{20}$ S. Hamaguchi, Phys. Fluids B 1, 1416 (1989).

${ }^{21}$ N. Nakajima, Phys. Fluids B 2, 1170 (1990).

${ }^{22}$ H. Sugama, N. Nakajima, and M. Wakatani, Phys. Fluids B 33290 (1991). 


\section{Figure Captions}

1. The contours of potential eigenfunctions given by Eq. (15). The quasilinear Reynolds stress increases the shear of the background poloidal flow for (a) $\alpha v_{E}^{\prime}>0$ and decreases it for $\alpha v_{E}^{\prime}<0$.

2. The linear growth rates for tiie resistive $g$-mode under the background poloidal flow given by the external one [Eq. (28)]. Three curves corresponds to different values of the maximum vorticity (or shear) $V_{0}^{\prime}: V_{0}^{\prime}=0$ (no background flow case), $V_{0}^{\prime}=0.1$ and $V_{0}^{\prime \prime}=0.2$.

3. The time evolution of the kinetic energy $\frac{1}{2} \int d x\left\langle v^{2}\right\rangle$ and the convective transport $\int d x\left\langle\tilde{p} \tilde{v}_{x}\right\rangle$ for $V_{0}^{\prime}=0.1$ and $\nu=0.02$.

4. The time evolution of the kinetic energy $\frac{1}{2} \int d x\left\langle v^{2}\right\rangle$ and the convective transport $\int d x\left\langle\tilde{p} \tilde{v}_{x}\right\rangle$ for $V_{0}^{\prime}=0.1$ and $\nu=0.3$.

5. Radial profiles of the convective flux $\left\langle\widetilde{p v}_{x}\right\rangle$ in the cases of Figs. 3 (a dashed line) and $\$$ (a solid line $)$.

6. Radial profiles of the background poloidal flow $v_{E}(x)$ and the vorticity $v_{E}^{\prime}(x)$ at the time $t=2000$ in the case of Fig. 3 . The dashed lines denote the external flow $V(x)$ and vorticity $V^{\prime}(x)$.

7. The contours of the $m=1$ mode of the electrostatic potential in the case of Fig. 3 . Here $(a)$ is for the growing state $(t=500)$ and $(b)$ for the saturated state $(t=2000)$.

8. The convective flux $\left\langle\widetilde{p v}_{x}\right\rangle$ and the background poloidal flow $v_{E}^{\prime}$ at the mode resonant surface $x=0$ for $\nu=0.02$ and $\nu=0.3$. The solid circles correspond to the case of $V_{0}^{\prime}=0.05$ and the open circles to the case of $V_{0}^{\prime}=0.1$. Also plotted is the convective 
transport in the case where the background flow is fixed to be zero. The dominant mode number in the saturated state is $m=2$ for no background flow and $V_{0}^{\prime}=0.05$ cases, and $m=1$ for $V_{0}^{\prime}=0.1$.

9. The convective flux, the background poloidal flow shear at $x=0$ and the maximum linear growth rate $\gamma$ for of $V_{0}^{\prime \prime}=0,0.02,0.05,0.1$. Here $\nu=0.02$. The dominant poloidal mode number in the saturated state is $m=2$ for $V_{0}^{\prime}=0,0.02,0.05$ and $m=1$ $\operatorname{for} I_{0}^{\prime \prime}=0.1$.

10. The time evolution of the kinetic energy and the turbulent transport. Hr:e $v_{E}=0$ for $0<t<1000$ and $v_{E}$ is given by solving Eq. (11) with $V_{0}^{\prime \prime}=0.4$ and $\nu=0.3$ for $t>1000$.

11. The rime evolution of the kinetic energy and the turbulent transport. Here $v_{E}=0$ for $0<t<1000$ and $v_{E}$ is given by solving Eq. (11) with $V_{0}^{\prime}=0.4$ and $\nu=0.02$ for $t>1000$.

2. The time evolution of the kinetic energev and the turbulent transport. Here $v_{E}=0$ for $0<t<1000$ and $v_{E}$ is given by solving Eq. (11) with no external flow $\left(V_{0}=0.0\right)$ and $z=0.02$ for $t>1000$. 
(a) $\alpha v_{E}^{\prime}>0$

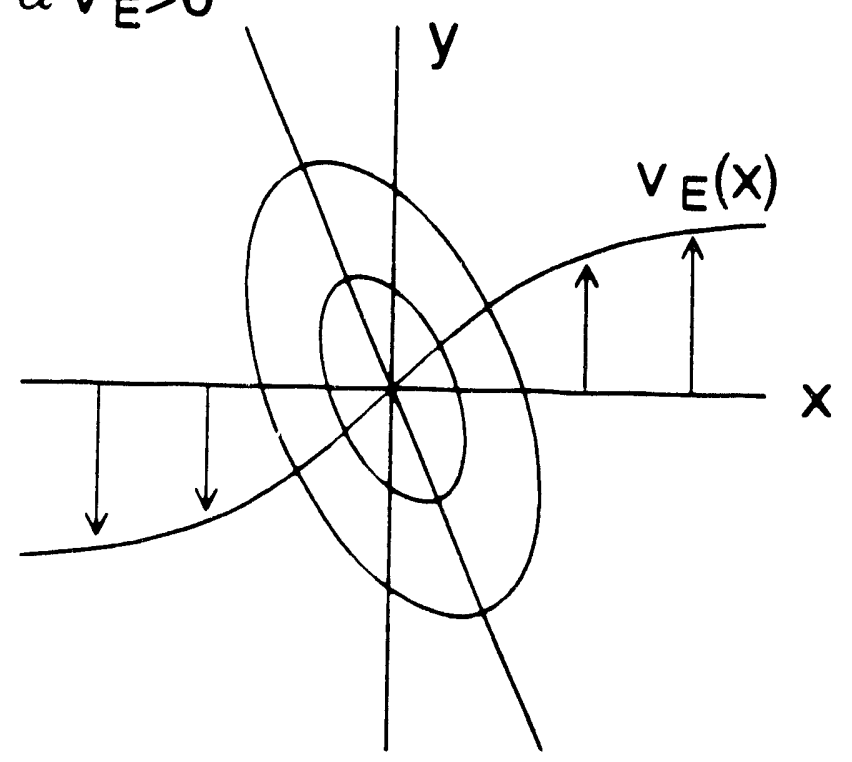

(b) $\alpha V_{E}^{\prime}<0$

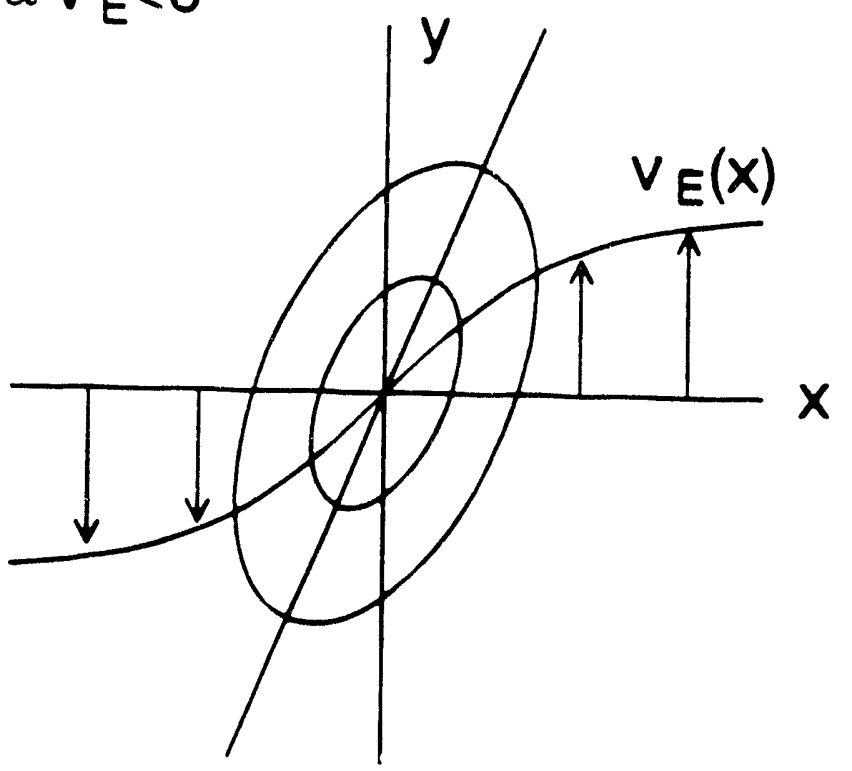

Fig.1 


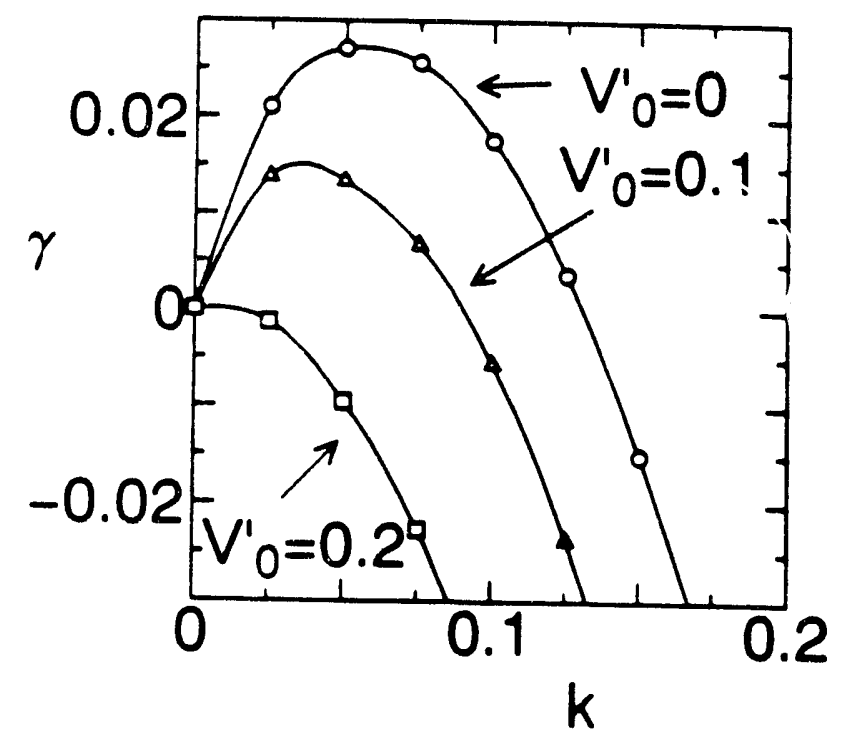

Fig.2 

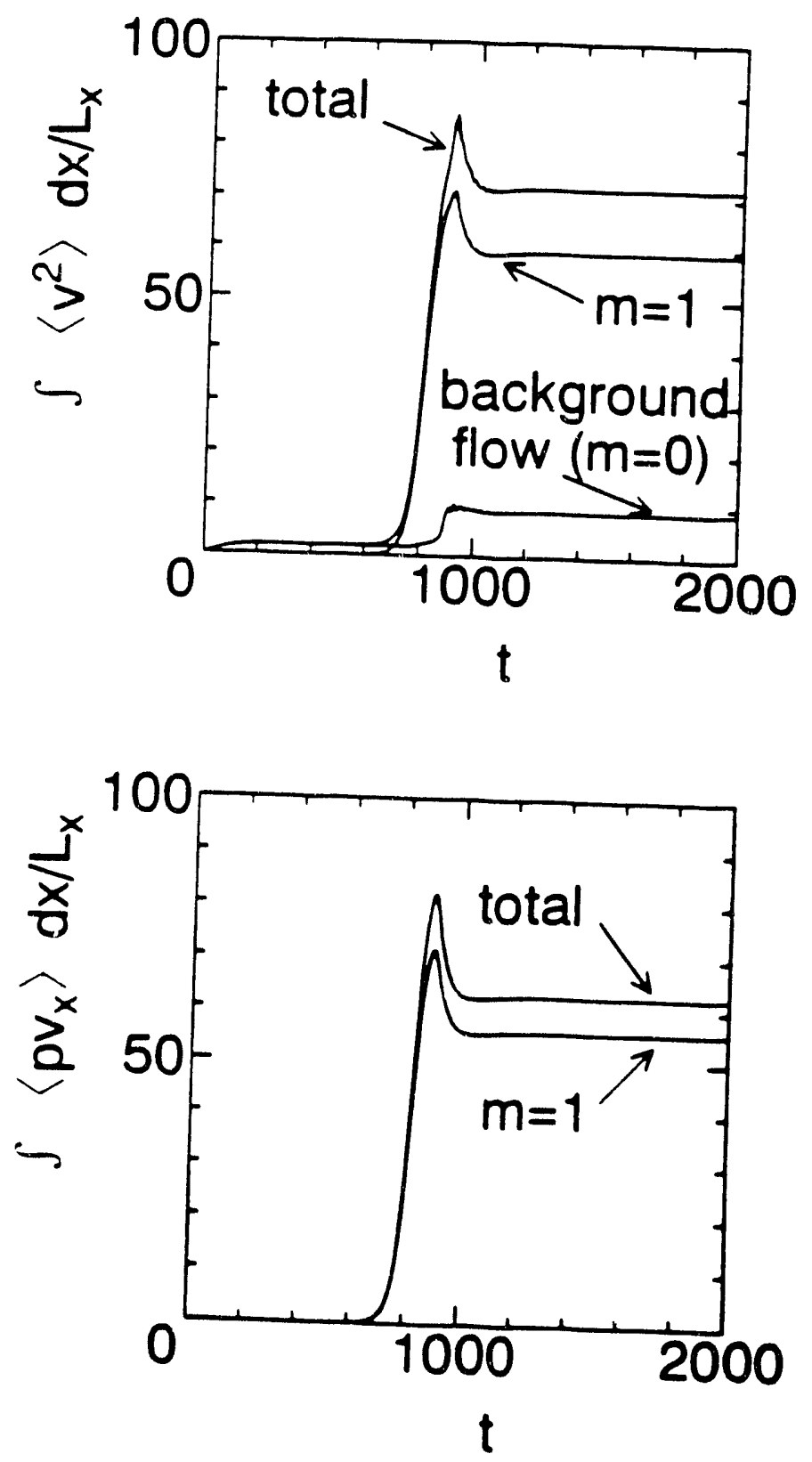

Fig.3 

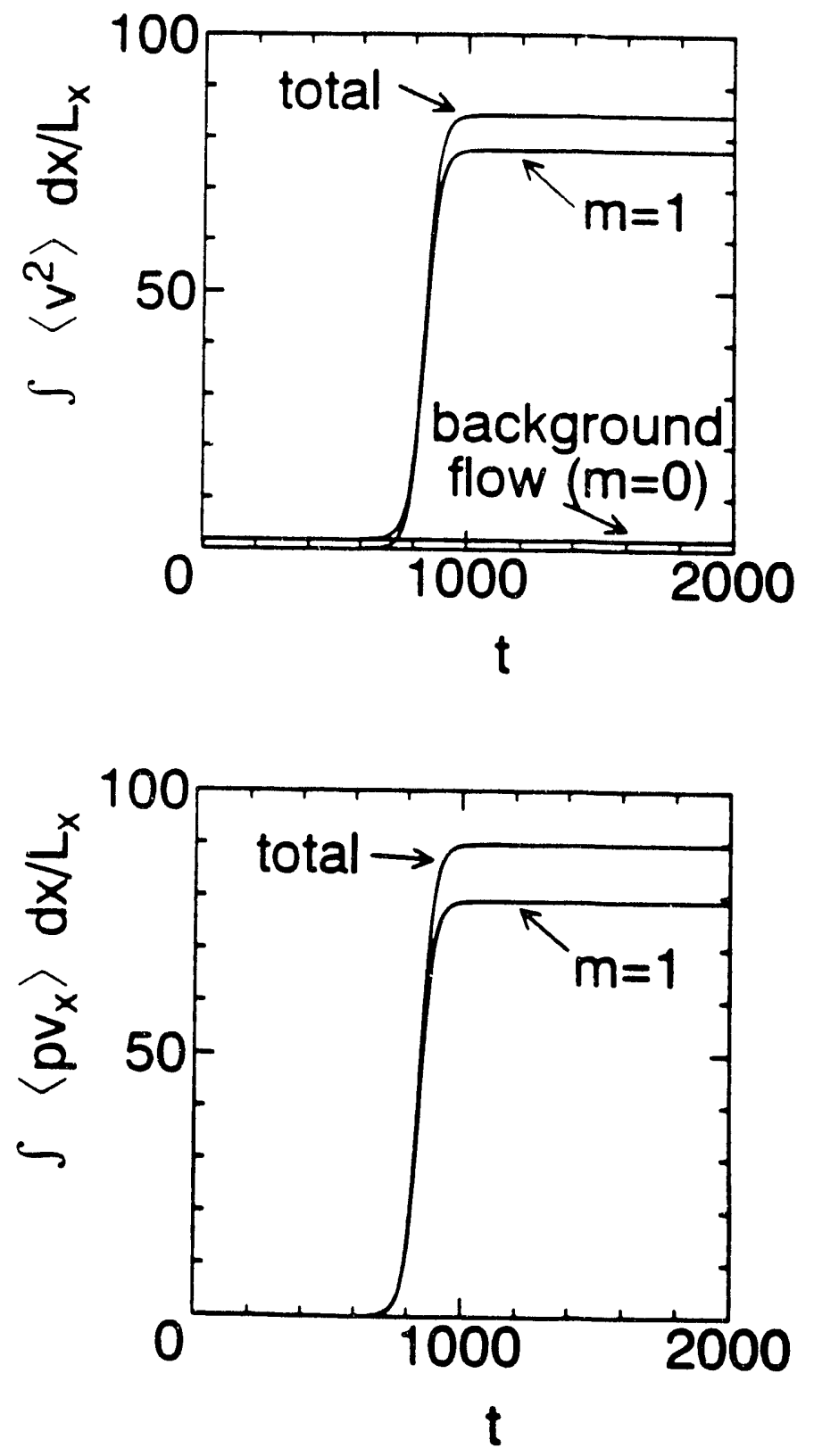

Fig.4 


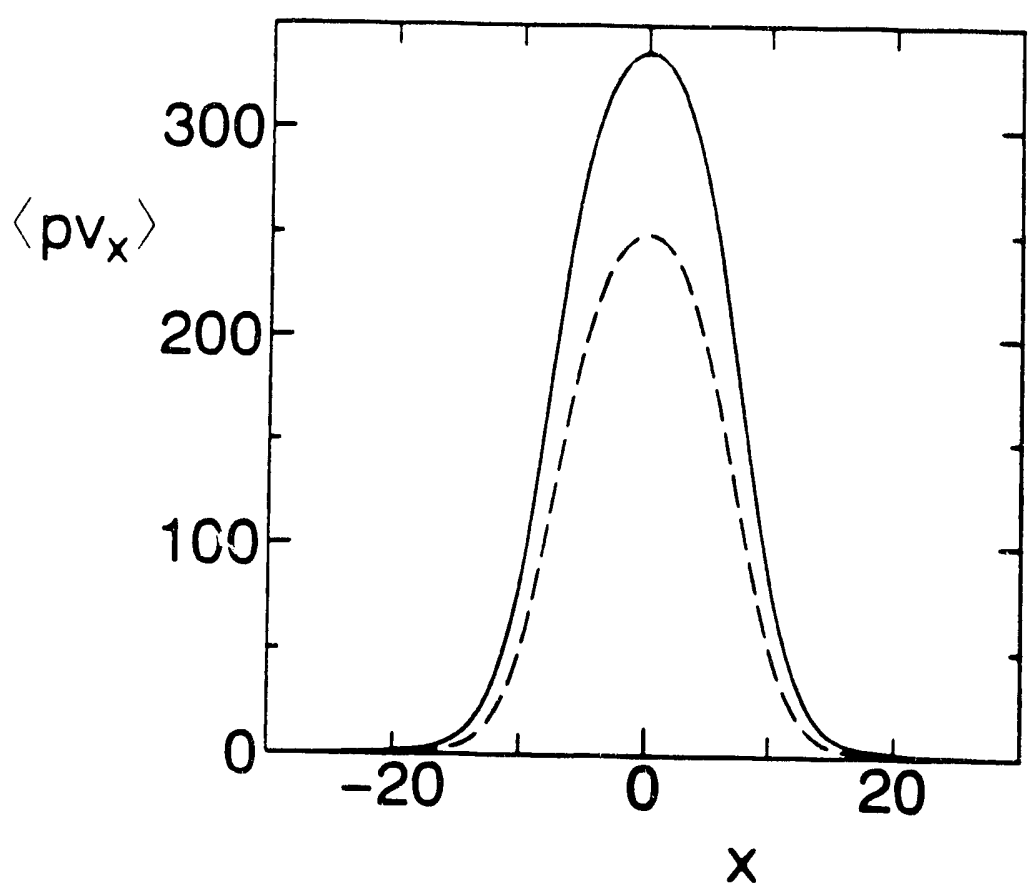

Fig.5 

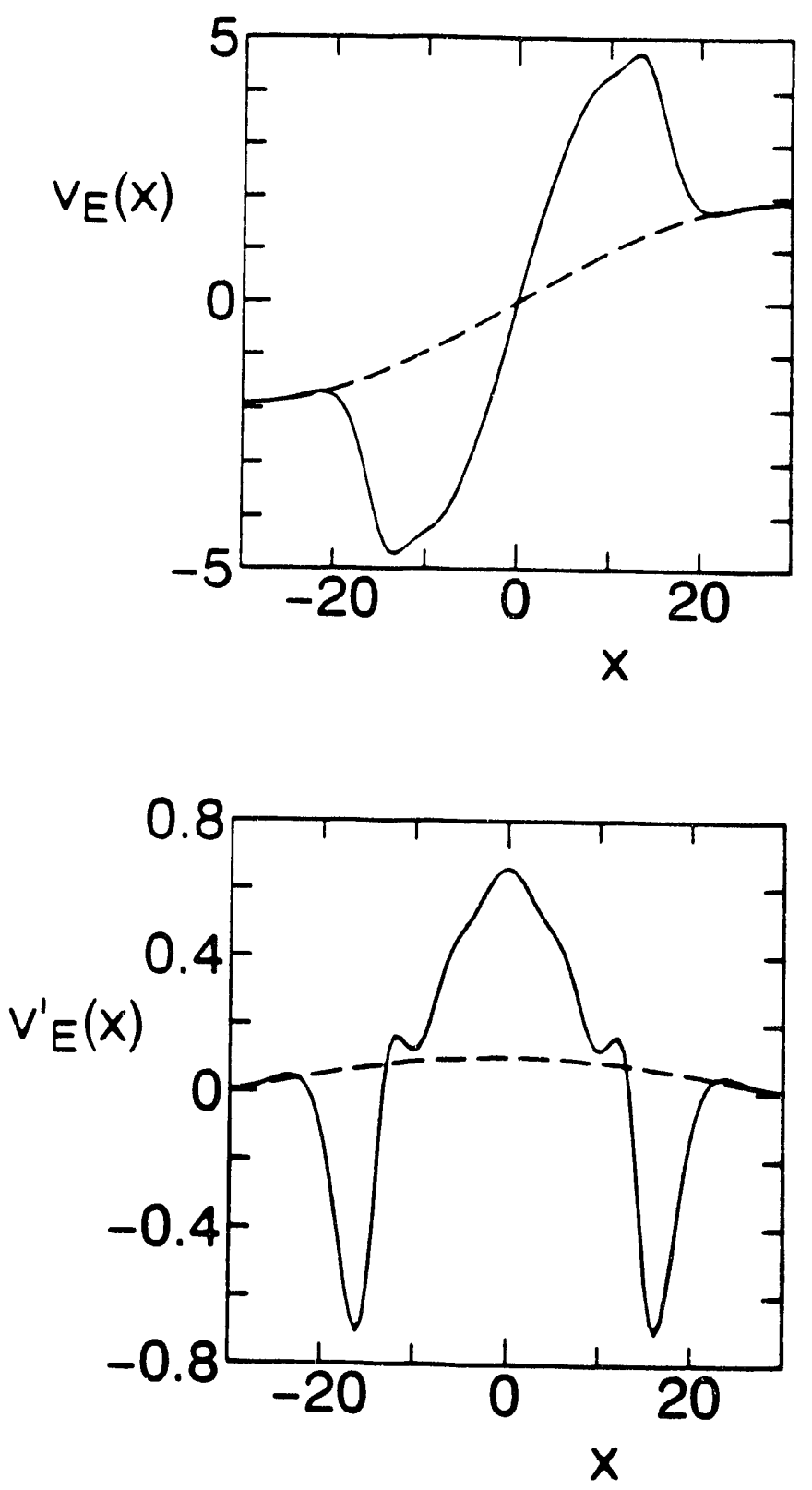

Fig.6 


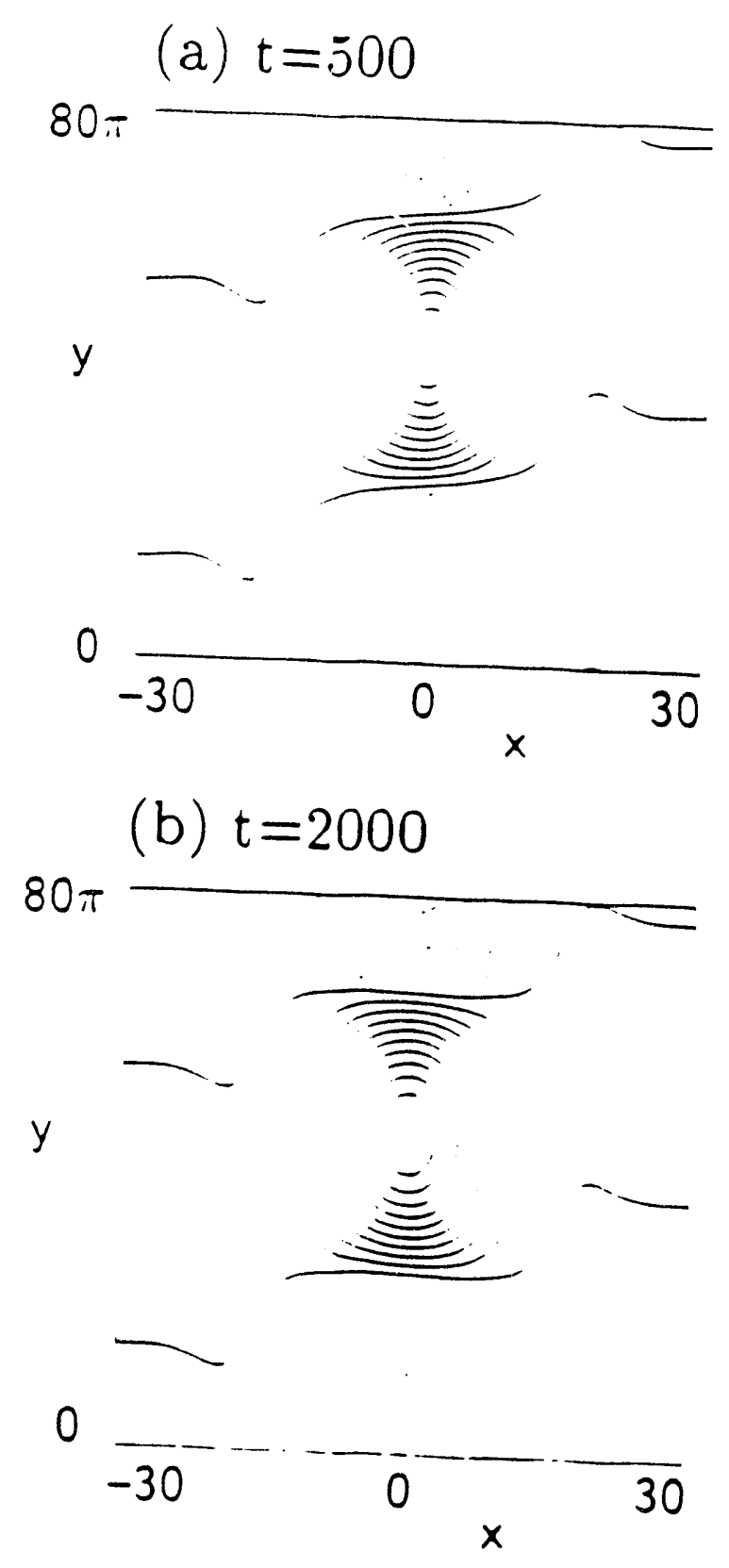

Fig. 7 


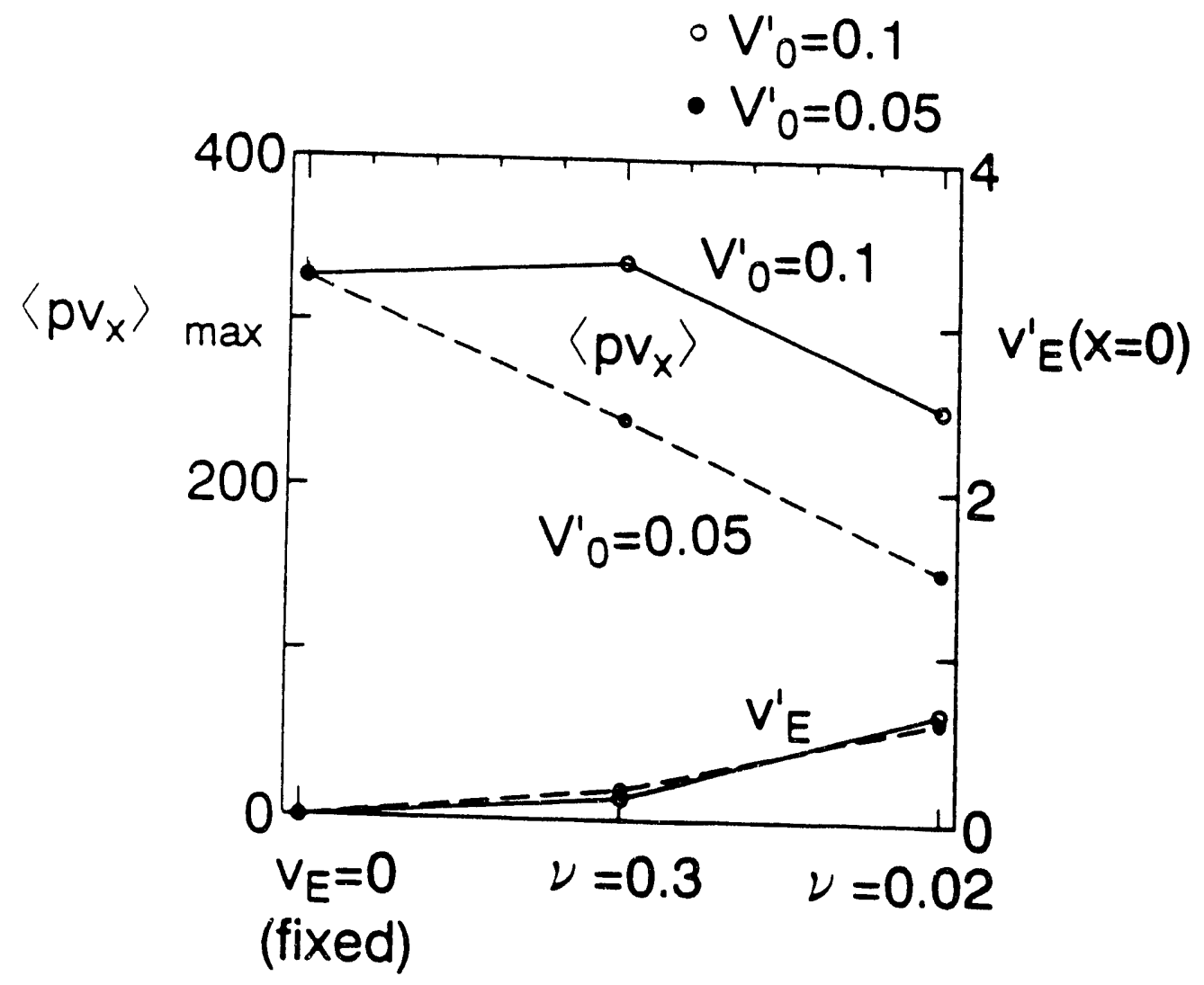

Fig.8 


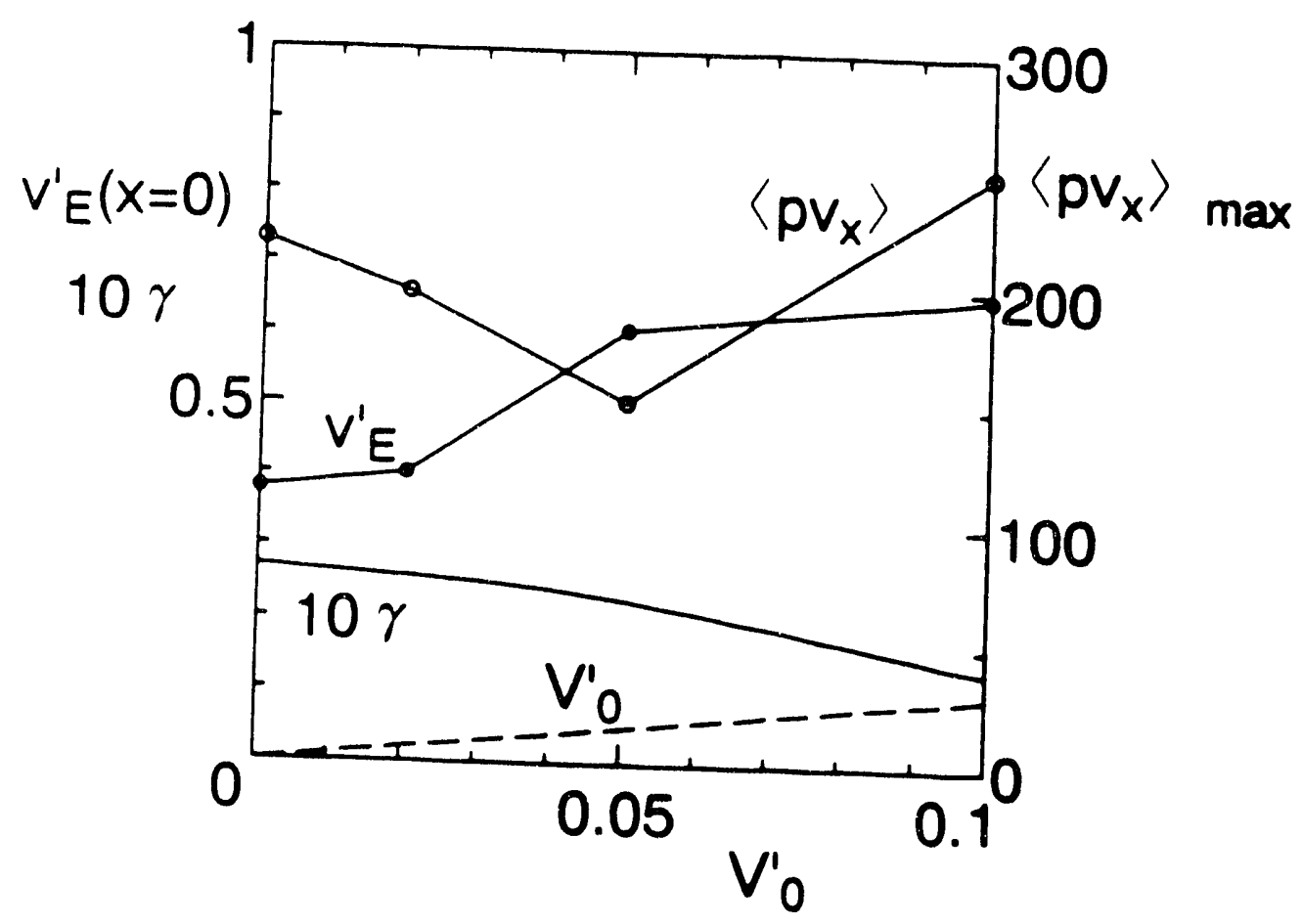

Fig.9 

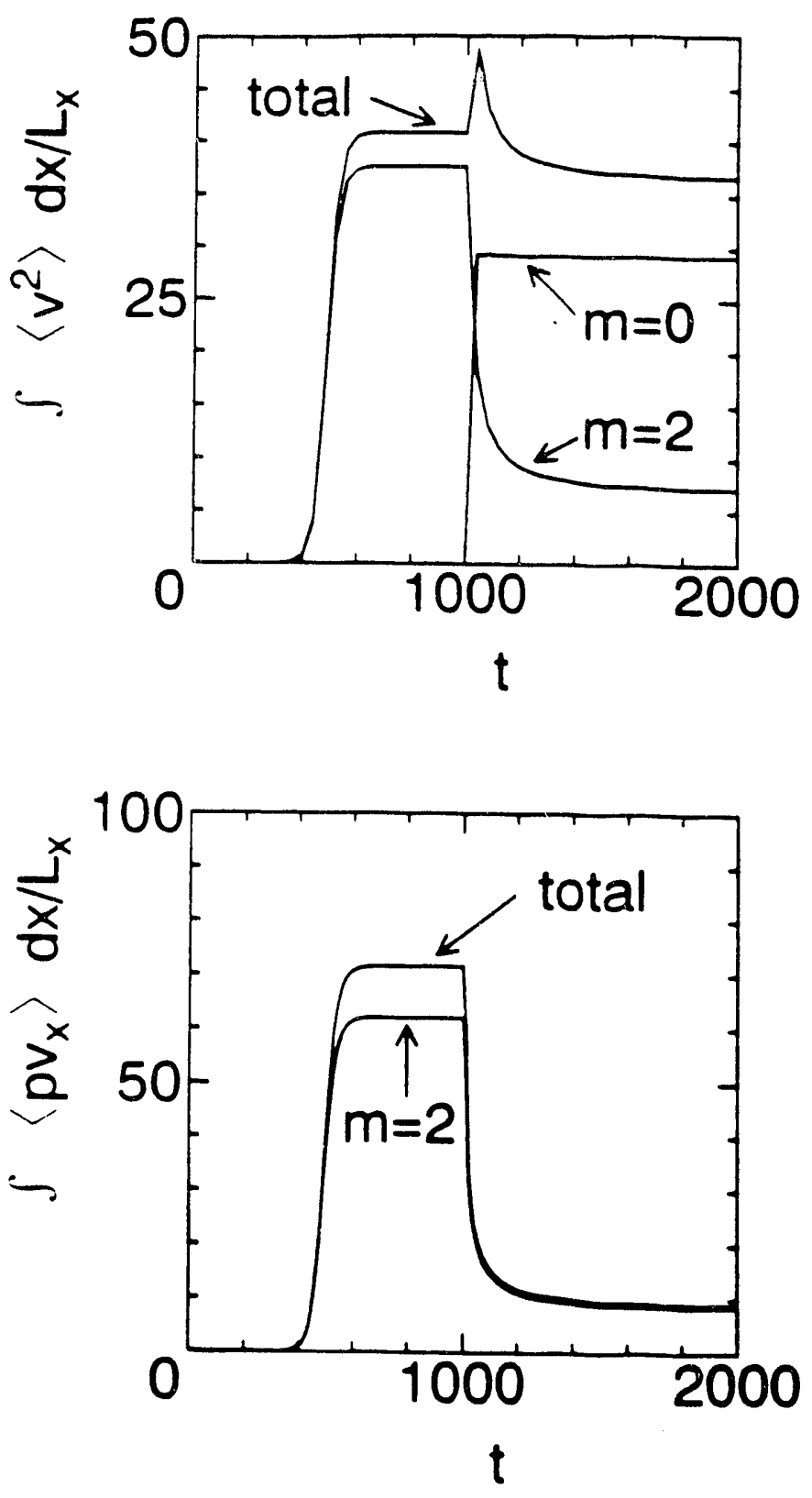

Fig.10 

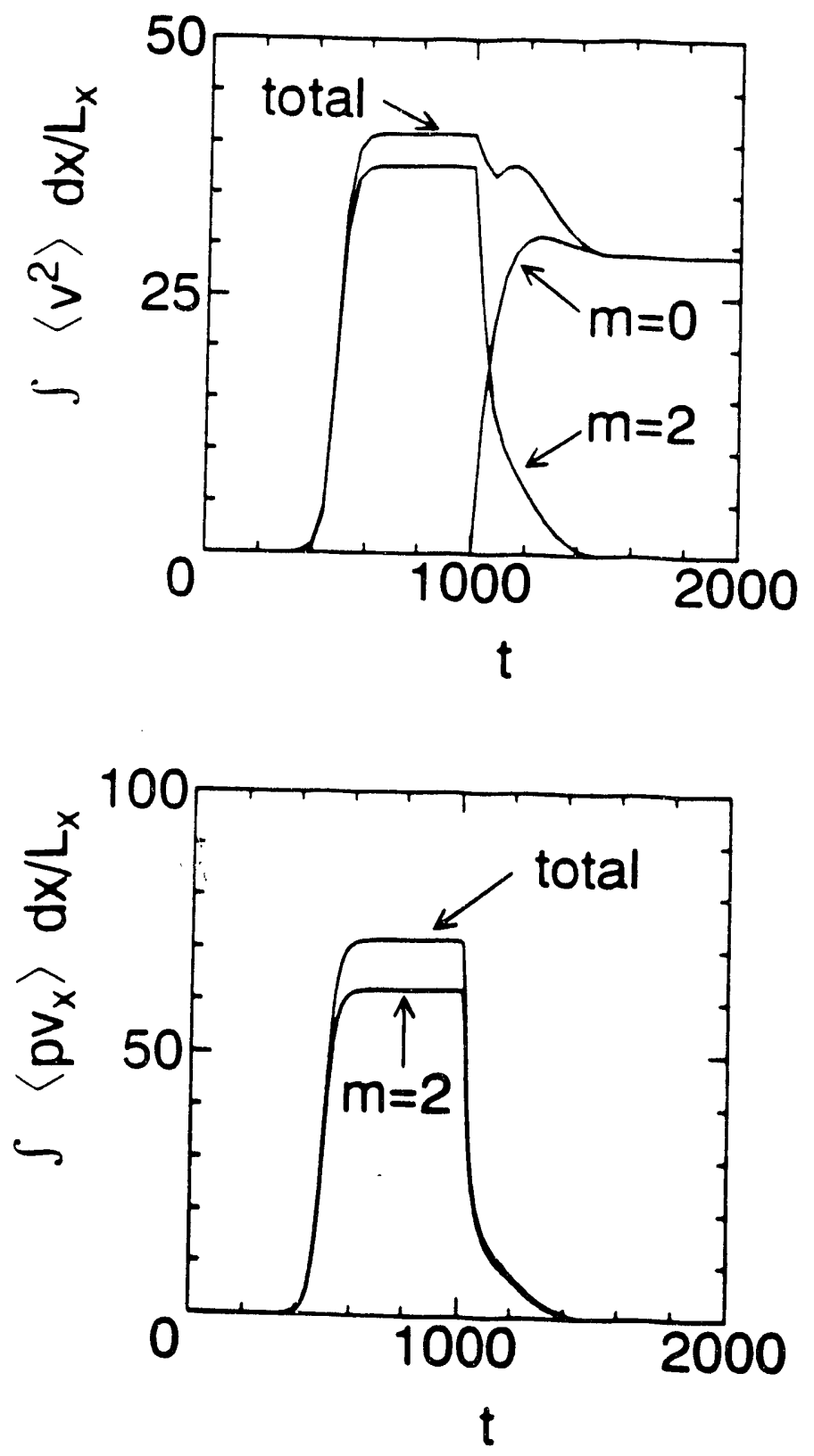

Fig.11 

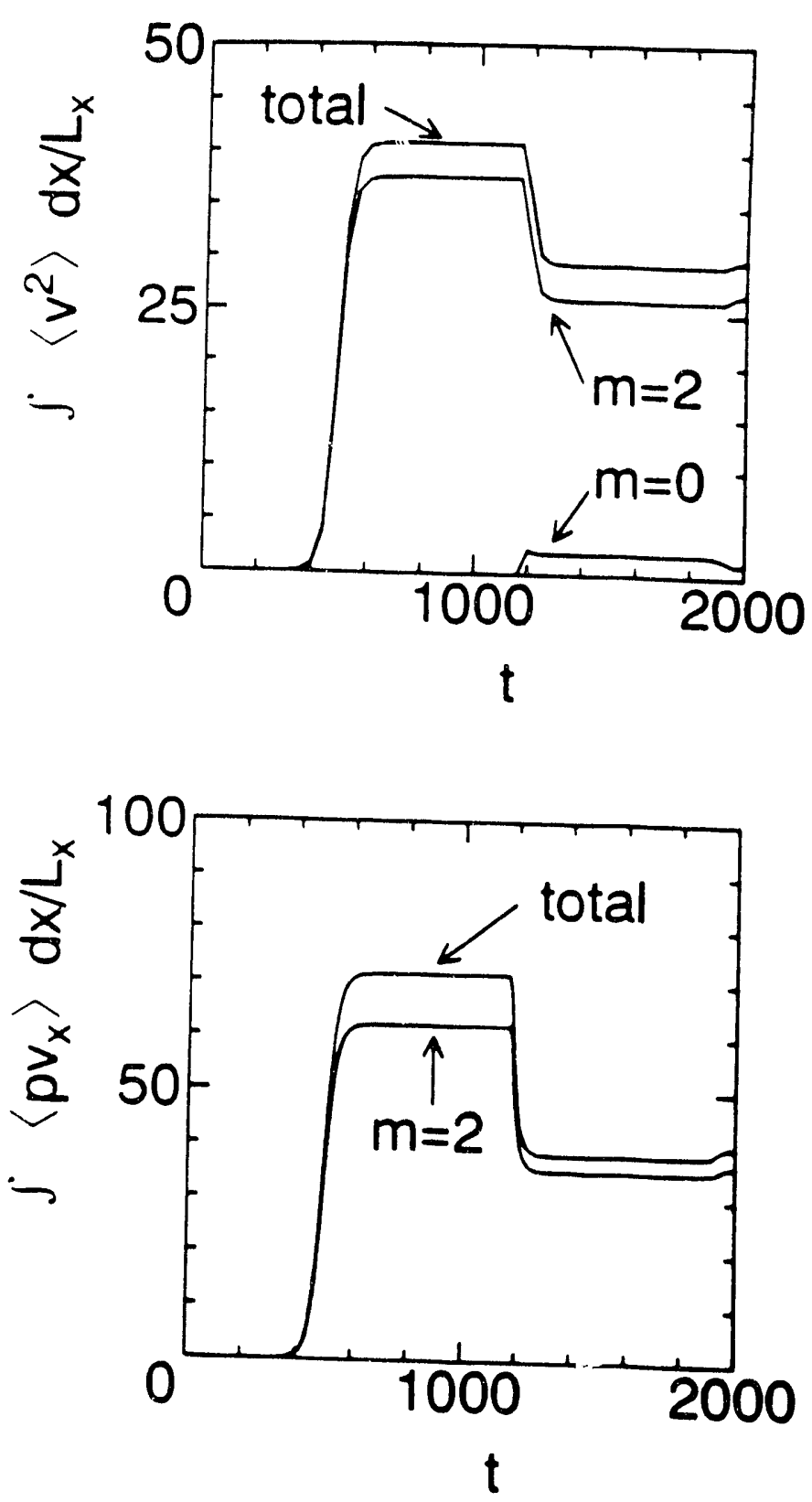

Fig.12 

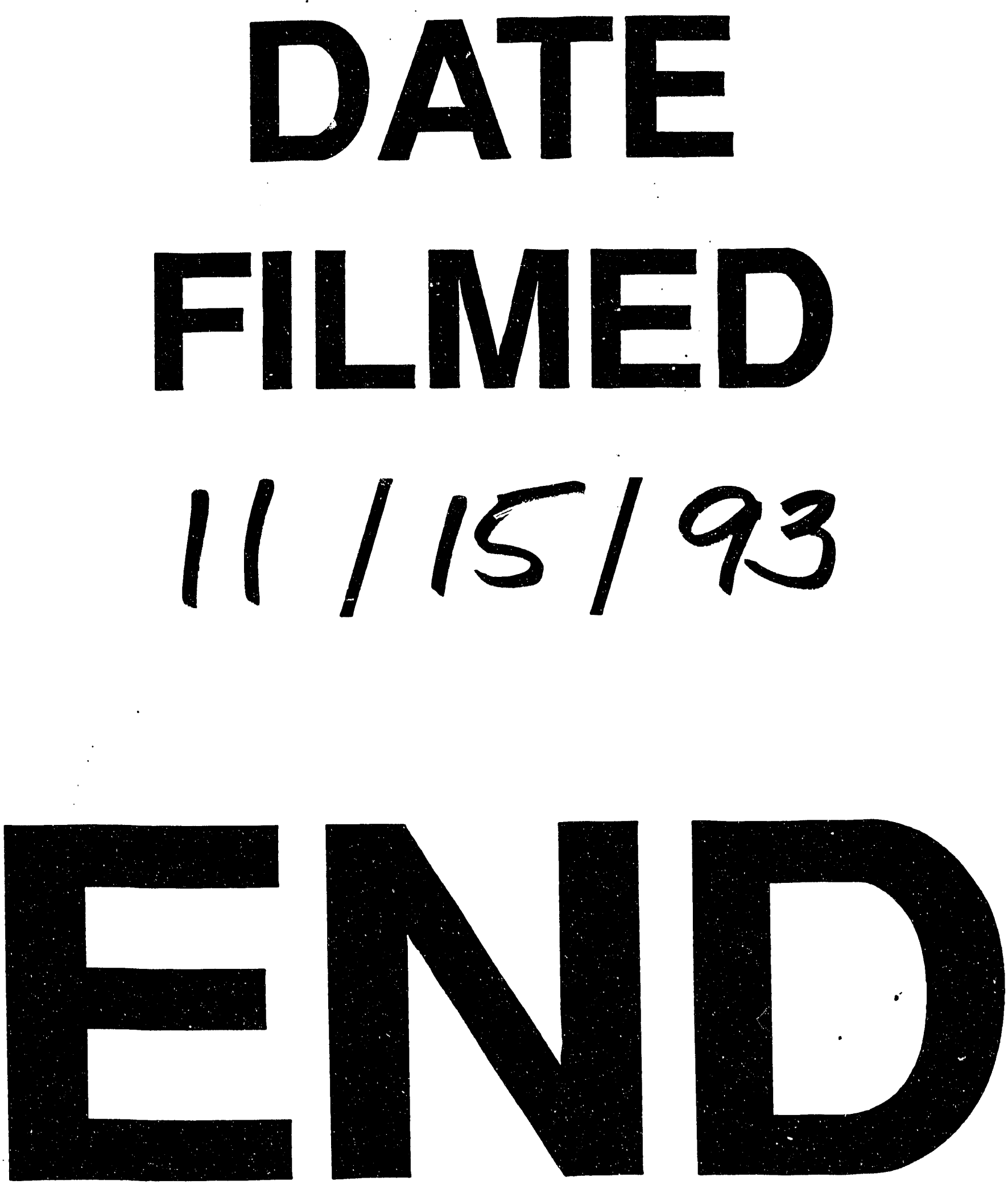